PAPER

\title{
Homocysteine and related genetic polymorphisms in Down's syndrome IQ
}

\author{
J-L Guéant*, G Anello*, P Bosco*, R-M Guéant-Rodríguez*, A Romano, C Barone, P Gérard, \\ C Romano
}

J Neurol Neurosurg Psychiatry 2005;76:706-709. doi: 10.1136/jnnp.2004.039875

See end of article for authors' affiliations

.....................

Correspondence to: Professor J L Guéant, EMI-INSERM 0014, Faculty of Medicine BP 184,54500 , Vandoeuvre lès Nancy, France; Jean-Louis.Gueant@ medecine.uhp-nancy.fr

Received 24February 2004 Revised version received 3 September 2004 Accepted

17 September 2004
Objective: Down's syndrome (DS) is the most frequent genetic cause of Alzheimer-type dementia. Its metabolic phenotype involves an increased trans-sulphuration of homocysteine. The aim of the present study was to investigate the influence of homocysteinaemia ( $\left.t-\mathrm{H}_{c y s}\right)$, folate, vitamin $B_{12}$, and related polymorphisms on intelligence quotient (IQ) in DS.

Methods: The IQ of 131 patients with trisomy 21 from a specialist centre in Sicily was determined and classified according to DMS-IV. The effects of age, folate, vitamin $\mathrm{B}_{12}, \mathrm{t}-\mathrm{Hcys}$, and genetic polymorphisms on IQ were evaluated separately and in combination using regression analyses.

Results: IQ was significantly lower in DS patients with $\mathrm{t}-\mathrm{H}$ cys $>7.5 \mu \mathrm{mol} / \mathrm{I}$ (median) and in those who were carriers of methylenetetrahydrofolate reductase (MTHFR) $677 \mathrm{~T}$ allele and of methylenetetrahydrofolate reductase $677 T$ and transcobalamin $776 \mathrm{G}$ combined alleles $(p=0.0013, p=0.0165$, and $p=0.0074$, respectively). The IQ correlated significantly with $\mathrm{t}-\mathrm{H}$ cys and folate in single and multiple regression analyses, independently of age. In addition, $\mathrm{t}-\mathrm{Hcys}>9.6 \mu \mathrm{mol} / \mathrm{l}$ (upper quartile) was found to be associated with low $I Q(<40$, median of study group) with an odds ratio of 2.61 ( $p=0.0203)$. The odds ratio was increased by threefold in carriers of MTHFR $677 T$ allele. The MTHFR $677 T$ allele/ transcobalamin $776 \mathrm{G}$ allele combination was associated with the risk of DS patients to have an IQ less that the median with an odds ratio of $2.68(95 \% \mathrm{Cl} 1.26$ to $5.70, p=0.0104)$.

Conclusion: This study found evidence of an association between t-Hcys and MTHFR $677 T$ and transcobalamin $776 \mathrm{G}$ alleles with $\mathrm{IQ}$ in patients with $\mathrm{DS}$. The association may be related to a defective remethylation of homocysteine, affecting $I Q$.
D own's syndrome (DS), or trisomy 21 , is the most frequent genetic cause of mental retardation. It results from the gene expression of an extra chromosome 21 , which occurs due to the failure of normal chromosomal segregation during meiosis. ${ }^{1}$ A genetic defect of the onecarbon metabolism may be associated with increased transsulphuration of homocysteine and chromosomal instability. ${ }^{2-4}$ The plasma homocysteine level (t-Hcys) is affected by folate, vitamin $B_{12}$, and by genetic polymorphism of three key enzymes of the one-carbon metabolism-methylenetetrahydrofolate reductase (MTHFR), methionine synthase (MTR; which catalyses the methyl transfer from methyltetrahydrofolate to t-Hcys), and methionine synthase reductase (MTRR; which maintains MTR in an active state). MTHFR $677 C \rightarrow T$ polymorphism is associated with elevated blood levels of t-Hcys, particularly in case of folate deprivation. ${ }^{56}$

Patients with DS develop dementia related to neocortex lesions similar to those of Alzheimer's disease (AD) - for example, senile plaques and neurofibrillary tangles. ${ }^{7}$ Some nutritional and genetic risk factors related to vascular diseases are associated with $\mathrm{AD}$. A strong association has been found with $\epsilon 4$ allele of apolipoprotein $E(A p o E),{ }^{8}$ and several studies have suggested a weaker association with $\mathrm{t}$-Hcys, folate, and vitamin $\mathrm{B}_{12}{ }^{9-12}$ but not with the MTHFR gene polymorphisms. ${ }^{13-15}$ Hyperhomocysteinaemia is also associated with impairment of cognitive function in elderly people. ${ }^{1016} 17$ The MTHFR $677 C \rightarrow T$ and MTRR $66 \quad A \rightarrow G$ polymorphisms are associated with a greater risk for mothers to have a child with DS. ${ }^{18} 19$ However, it remains unknown whether t-Hcys and its genetic and related nutritional determinants influence the intelligence quotient (IQ) in DS patients.
In the present study, we investigated the relations between IQ, blood level of t-Hcys, related vitamins (folate and vitamin $\left.\mathrm{B}_{12}\right)$, and genotypes of MTHFR, MTR, MTRR, transcobalamin $(T C N)$, in 131 patients with DS.

\section{PATIENTS AND METHODS \\ Patients}

The 131 outpatients were recruited after obtaining the family's and patient's informed consent in a specialist centre receiving patients only from Sicily. The centre's ethical committee approved the study.

Karyotyping showed full trisomy 21 in $100 \%$ of patients. The clinical characteristics taken into account when analysing the data were body mass index (BMI; only for patients older than 10 years), coeliac disease, cardiopathy, thyroid status, and epilepsy. None of the patients smoked, had renal failure (creatinine $<15 \mathrm{mg} / \mathrm{l}$ in all cases), or took vitamin supplements or food fortified with vitamins. In all patients, the thyroid status was either normal or rendered euthyroid by replacement therapy. The IQ was tested with the Wechsler scales for adults and children, as described previously. ${ }^{20} 21$ After testing, all subscale scores were transformed into agescaled scores, the standard IQ calculated, and the mental retardation classified according to the DSM-IV, as specified by the American Society of Psychiatry (2000). ${ }^{22}$

Abbreviations: AD, Alzheimer's disease; ApoE, Apolipoprotein E; BMI, body mass index; DS, Down's syndrome; IQ, intelligence quotient; MTHFR, methylenetetrahydrofolate reductase; MTR, methionine synthase; MTRR, methionine synthase reductase; $t-H_{c y s}$, homocysteine; $\mathrm{TCN}$, transcobalamin 
Table 1 Influence of homocysteine and genetic polymorphisms of methylenetetrahydrofolate reductase (MTHR), methionine synthase (MTR), methionine synthase reductase (MTRR), transcobalamin (TC) and apolipoprotein $E$ (ApoE) on intelligence quotient (IQ; median (25th-75th centile)) in patients with Down's syndrome

\begin{tabular}{|c|c|c|c|c|}
\hline \multirow[b]{2}{*}{ Characteristic } & \multirow[b]{2}{*}{ n (\%) } & \multicolumn{2}{|l|}{$\mathbf{I Q}$} & \multirow[b]{2}{*}{ p value* } \\
\hline & & Yes & No & \\
\hline Homocysteine $>7.5 \mu \mathrm{mol} / /$ (median) & $66(50.3)$ & $35(25-45)$ & $45(35-55)$ & 0.0013 \\
\hline Homocysteine $>9.6 \mu \mathrm{mol} / /$ (75th centile) & $34(25.9)$ & $35(20-45)$ & $40(35-50)$ & 0.0021 \\
\hline MTHFR $677 \mathrm{~T}$ allele & $85(65.4)$ & $35(30-46)$ & $45(35-55)$ & 0.0165 \\
\hline MTHFR 1298 C allele & $76(58.0)$ & $40(32-50)$ & $35(25-50)$ & 0.2762 \\
\hline MTR 2756 G allele & $52(40.0)$ & $40(32-50)$ & $35(30-50)$ & 0.2849 \\
\hline TCN 776 G allele & $95(73.0)$ & $35(30-50)$ & $40(35-50)$ & 0.1895 \\
\hline ApoE $\epsilon 4$ allele & $15(11.7)$ & $45(36-55)$ & $40(30-50)$ & 0.1039 \\
\hline MTHFR 677 T allele/MTHFR 1298 C allele & $39(30.0)$ & $40(30-45)$ & $40(30-50)$ & 0.4251 \\
\hline MTHFR 677 T allele/MTR $2756 \mathrm{G}$ allele & $32(24.6)$ & $40(30-50)$ & $39(30-56)$ & 0.9483 \\
\hline MTHFR 677 T allele/TCN $776 \mathrm{G}$ allele & $63(48.5)$ & $35(28-45)$ & $45(35-54)$ & 0.0074 \\
\hline MTHFR $677 T$ allele/ApoE $\epsilon 4$ allele & $10(7.8)$ & $42(35-45)$ & $40(30-50)$ & 0.6222 \\
\hline
\end{tabular}

\section{Laboratory assays}

Blood was collected in the fasting state. Plasma t-Hcys was assayed by fluorescence polarisation immunoassay (FPIA), and vitamin $\mathrm{B}_{12}$ and folate by microparticle enzyme immunoassay (MEIA) using the Abbott IMx automated benchtop analyser system (Abbott Diagnostic, Rome, Italy). DNA was isolated from a lymphocyte enriched fraction of whole blood with NUCLEON BACC3 for extraction of genomic DNA kit (Amersham Pharmacia Biotech, Milan, Italy). The procedures for detecting the $677 C \rightarrow T$ and 1298 $A \rightarrow C$ polymorphisms of MTHFR, as well as the $2756 A \rightarrow G$ MTR and the $66 A \rightarrow G$ MTRR polymorphisms, were based on polymerase chain reaction (PCR) amplification, restriction cleavage and separation of the DNA fragments by 15\% nondenaturant polyacrylamide gel electrophoresis (SDS-PAGE), as previously described..$^{18}$ The TCN $776 C \rightarrow G$ polymorphism was genotyped by the amplification refractory mutation system, as described recently by us. ${ }^{23}$ DNA samples corresponding to amplified DNA of the MTHFR, MTR, and $M T R R$ genotypes were sequenced and subsequently used as controls in all series of genotype determination.

\section{Statistical methods}

Categorical variables are reported as counts and percentages, and continuous variables as median, 25th and 75th centiles. For categorical and continuous variables, a continuity corrected $\chi^{2}$ test and the Mann-Whitney $U$ test were used, respectively. Spearman's rank correlation coefficient was used to estimate the correlation among IQ, t-Hcys, age, folate and vitamin $\mathrm{B}_{12}$. The significance and odds ratios of continuous and categorical variables regarding mental retardation were determined by stepwise multiple regression and logistic regression analyses, respectively. A p value higher than 0.10 was set to exclude variables in the stepwise analyses and a final $\mathrm{p}$ value lower than 0.05 was considered to indicate residual statistical significance. Data were collected and analysed using the Statview 5 software for
Windows (SAS Institute, Berkley, CA, USA) and the SPSS 10.0 software for Windows (SPSS, Paris, France).

\section{RESULTS}

The IQ of the 131 patients with DS ranged from 70 to 10, with median, 25th and 75th centiles of 40,30, and 60, respectively. The plasma homocysteine levels were close to those observed in a control population (median, 25th and 75th centiles 7.5, 5.7, and $9.6 \mu \mathrm{mol} / \mathrm{l}$, respectively). The plasma levels exceeded the $15 \mu \mathrm{mol} / \mathrm{l}$ limit of moderate hyperhomocysteinaemia in nine patients, including five with a severe (IQ 35-25) and two with a profound $(\mathrm{IQ}<25)$ degree of mental retardation, according to the four degrees of the DMS-IV classification. ${ }^{22}$ We also found that patients who had t-Hcys levels $>7.5 \mu \mathrm{mol} / \mathrm{l}$ had an IQ significantly lower than that of those who had a concentration below this median value $(p=0.0013$, table 1$)$. Reciprocally, $t$-Hcys was higher in DS patients with an IQ $<40$ (median of the study group), compared with those with less mental retardation (median and interquartiles: 8.19, 6.20, 10.50 and 6.80, 5.37, $8.70 \mu \mathrm{mol} / \mathrm{l}$, respectively; $\mathrm{p}=0.004)$.

Since t-Hcys was also related to age (table 2), we performed the same analysis in two age matched subgroups with IQ $<40$ ( $\mathrm{n}=29$, mean (SD) age 19.7 (9.3) years) and $>40(\mathrm{n}=40$, mean age 20.3 (8.8) years). The t-Hcys plasma level was still higher in the age matched group with IQ $<40$ (median and interquartiles: 8.4, 7.1, 9.3 and 6.9, 5.4, $8.8 \mu \mathrm{mol} / \mathrm{l}$, respectively; $\mathrm{p}=0.005)$. The IQ correlated significantly with t-Hcys and folate in log rank Spearman's univariate analysis (table 2). This association was seen mainly in the patients who had the lowest IQ $(<$ median) (see table 2). Conversely, a significant correlation was found between IQ and age, mostly in the patients with the less severe degrees of mental retardation. Indeed, this correlation was not significant when only patients with IQ $<40$ were considered (table 2). In a stepwise multiple regression analysis that also included vitamin $\mathrm{B}_{12}$, transcobalamin,

Table 2 Log rank Spearman's correlation $\left(r_{s}\right)$ between intelligence quotient (IQ) and either age, homocysteine, or folate in 131 patients with Down's syndrome (DS) and in two subgroups divided by IQ median $(I Q=40)$

\begin{tabular}{|c|c|c|c|c|c|c|c|}
\hline \multirow[b]{2}{*}{ Group } & \multirow[b]{2}{*}{ No of cases } & \multicolumn{2}{|l|}{ Age } & \multicolumn{2}{|c|}{ Homocysteine } & \multicolumn{2}{|l|}{ Folate } \\
\hline & & $r_{s}$ & $p$ value & $r_{\mathrm{s}}$ & $p$ value & $r_{s}$ & p value \\
\hline $\begin{array}{l}\text { All DS } \\
I Q \geqslant 40 \\
\mid Q<40\end{array}$ & $\begin{array}{r}131 \\
69 \\
62\end{array}$ & $\begin{array}{l}-0.455 \\
-0.546 \\
-0.101\end{array}$ & $\begin{array}{l}<0.0001 \\
<0.0001 \\
0.4285\end{array}$ & $\begin{array}{l}-0.329 \\
-0.167 \\
-0.269\end{array}$ & $\begin{array}{l}0.0002 \\
0.1689 \\
0.0355\end{array}$ & $\begin{array}{l}0.110 \\
0.088 \\
0.328\end{array}$ & $\begin{array}{l}0.2111 \\
0.4705 \\
0.0104\end{array}$ \\
\hline
\end{tabular}


thyroid stimulating hormone (TSH), BMI, and age, the IQ remained associated only with t-Hcys (initial and residual $\mathrm{p}$ values 0.0112 and 0.0037 , respectively) and folate (initial and residual p values 0.0967 and 0.0359 , respectively). In a stepwise logistic regression analysis, we found an association between t-Hcys $>9.6 \mu \mathrm{mol} / \mathrm{l}$ and IQ $<40$ (median) with an odds ratio of 2.61 (95\% confidence interval (CI) 1.16 to 5.88 ; $\mathrm{p}=0.0203$ ), and IQ $<30$ (10th centile) with an odds ratio of 3.21 (95\% CI 1.40 to $7.37, \mathrm{p}=0.0057$ ).

There was no difference with regard to t-Hcys, folate, and vitamin $B_{12}$ between patients with coeliac disease and the other patients $(p=0.363,0.720,0.351$, respectively). We investigated the independent determinants of $\mathrm{t}$-Hcys by multiple regression analysis-age and folate were but vitamin $\mathrm{B}_{12}$, transcobalamin, TSH, and BMI were not significant determinants $(p=0.0034$ and $p=0.0232$, respectively).

The distributions of MTHR, MTR, MTRR, and TCN genotypes were in Hardy-Weinberg equilibrium. We evaluated the influence of these polymorphisms on the IQ, both alone and in combination with each other. The patient with DS bearing the MTHFR 677 T allele and the MTHFR 677 T/TCN $776 \mathrm{G}$ allele combination had an IQ significantly lower than those carrying the corresponding wild genotypes (table 1). This effect was not age related, as the MTHFR $677 \mathrm{~T}$ allele and the MTHFR 677 T/TCN $776 \mathrm{G}$ allele combination had no influence on the age of carriers $(\mathrm{p}=0.6299$ and $\mathrm{p}=0.7426$, respectively). We also found a significant association of the MTHFR $677 \mathrm{~T}$ allele/TCN $776 \mathrm{G}$ allele combination with the risk of DS patients to have a low IQ $<$ median with an odds ratio of 2.68 (95\% CI 1.26 to $5.70, p=0.0104)$. There was no direct association of MTHFR $677 \mathrm{~T}$ allele with the risk of low IQ. However, this genotype increased the risk of low IQ associated with t-Hcys by about threefold with an odds ratio of 7.78 (95\% CI 11.20 to $50.43, \mathrm{p}=0.0315)$.

\section{DISCUSSION}

Several clinical and experimental studies have hypothesised that patients with DS have disturbed one-carbon metabolism. ${ }^{2}{ }^{19}{ }^{25-27}$ t-Hcys, vitamin $B_{12}$, and folate are metabolic and nutritional factors directly related to this metabolism. However, it remained unknown whether these factors and the associated genetic polymorphisms aggravate the age related mental retardation in DS.

The relatively low blood levels of t-Hcys in our patients can possibly be explained by an overexpression of the chromosome 21 cystathionine- $\beta$-synthase enzyme, as has been observed by others. ${ }^{28}$ Despite these relatively low levels, we found a significant association between IQ and t-Hcys. Several hypotheses may explain this association. The relatively high t-Hcys levels in the subgroup of patients who had the lowest IQ could be an indirect consequence of mental retardation, since the latter may lead to reduced autonomy and subsequent deficient dietary intake. In fact, in our study, the influence of diet seemed to be limited to folate. We compared two age matched groups consisting of outpatients from an socioeconomic background. The BMI had no influence on the IQ average, and a significant correlation of IQ with folate was observed in only half of the patients with DS - those who had the highest level of mental retardation (see table 2). In addition, multiple regression analysis showed that neither vitamin $\mathrm{B}_{12}$ nor BMI were independent determinants of t-Hcys, and that folate was a weak determinant. Age is another factor that may be involved in the association between IQ and t-Hcys; it is known to be a determinant of both t-Hcys and cognitive function. ${ }^{16}$ However, our study suggests that age alone cannot explain the t-Hcys/IQ association in DS. Indeed, the influence of age on IQ was observed in only half the patients, those who had the lowest level of mental retardation. In addition, IQ was associated with t-Hcys independently of age in the multiple regression analysis.

We also investigated the role of genetic determinants of $\mathrm{t}$-Hcys in the association between t-Hcys and IQ level. Carriers of the MTHFR $677 \mathrm{~T}$ allele had a lower IQ than those carrying the corresponding wild genotype (see table 1). This can be explained at least in part by its effect on the association of t-Hcys level with IQ since it increased the odds ratio of this association by threefold. The influence on IQ became slightly more significant when we considered the combination of MTHFR 677 T and TCN 776 G allele. TCN 776 $C \rightarrow G$ polymorphism has been reported to be a weak determinant of homocysteine in some but not all studies in healthy populations. ${ }^{232930}$ It may influence the cellular availability of vitamin $\mathrm{B}_{12}$ because it is associated with a decreased concentration of transcobalamin, the carrier protein which delivers vitamin $\mathrm{B}_{12}$ to cells, and with an increased blood level of methylmalonic acid. ${ }^{23}{ }^{31}$ The influence of MTHFR $677 \mathrm{~T}$ allele either alone or in combination with TCN $776 \mathrm{G}$ allele on IQ may correspond, therefore, to decreased remethylation of homocysteine due to reduced activity of MTHFR, eventually accentuated by reduced availability of vitamin $\mathrm{B}_{12}$, cofactor of MTR. None of the other genetic polymorphisms of the one-carbon metabolism influenced the level of mental retardation.

The trisomy 21 phenotypic abnormalities of one-carbon metabolism could theoretically be caused by the overexpression of genes on chromosome $21 .{ }^{32}{ }^{33}$ From this point of view, the increase in the activity of the trans-sulphuration pathway of t-Hcys, which results from the overexpression of cystathionine $\beta$-synthase on chromosome 21, 28 323337 may promote a "folate trap" by decreasing the cellular concentration of homocysteine and therefore its subsequent methyltetrahydrofolate and vitamin $\mathrm{B}_{12}$ dependent remethylation and the cellular synthesis of tetrahydrofolate. ${ }^{25} 28$ Indeed, supplementation with either folinic acid or methyl- $\mathrm{B}_{12}$ is very effective in increasing the defective cellular level of methionine and $S$-adenosylmethionine in lymphoblastoid cells of trisomy $21 .{ }^{25}$ In this metabolic context, a decreased activity of MTHFR, resulting from a 677 TT genotype, may act as an aggravating factor of the "folate trap" by decreasing the remethylation of homocysteine and the synthesis of tetrahydrofolate. The reduced activity of mutated MTHFR also decreases the level of $S$-adenosylmethionine, ${ }^{34}$ a substrate needed for pathways possibly involved in DS pathogenesis such as DNA and protein methylation and synthesis of choline. ${ }^{625}$ This polymorphism has been also described as a key genetic determinant of a folate imbalance between DNA synthesis on one hand and remethylation of homocysteine and DNA methylation on the other hand, which is effective only in the absence of folate repletion. ${ }^{6}{ }^{34}$ The hypothesis of a link between DNA methylation and cognitive dysfunction should be further investigated in the light of the present association of t-Hcys with IQ because t-Hcys correlates significantly with genomic DNA methylation. ${ }^{35}$

Recently, a follow up study showed that t-Hcys was an independent risk factor for the occurrence of dementia of sporadic $\mathrm{AD} .^{36} \mathrm{t}$-Hcys interacts with several pathomechanisms of sporadic AD. It impairs DNA repair in hippocampal neurones, promotes apoptosis, hypersensitivity to exitotoxicity and oxidative stress and it potentiates the neurone toxicity of $\beta$-amyloid peptide, the proteolytic product of amyloid precursor protein. ${ }^{37} 38$ In addition to the overexpression of cystathionine $\beta$-synthase, another phenotypic abnormality related to chromosome 21 genes is the overexpression of amyloid precursor protein, which confers a predominant role to the pathway of amyloid precursor protein in the neurodegeneration in DS dementia. ${ }^{73}$ It has 
been showed that homocysteine and folate deficiency increases the risk of neurodegenerative disorders. ${ }^{37}$ We also reported recently that MTR had an influence on the progression of $\mathrm{AD}$, which may be enhanced by carriage of allele $\epsilon 4$ of $A p o E .^{39}$ Therefore, our previous results and the present data argue for investigating the potential interactions between homocysteine and $\beta$-amyloid fragment metabolism in the pathogenesis of DS dementia.

\section{Authors' affiliations}

G Anello, P Bosco, C Barone, C Romano, IRCCS, Oasi Maria SSInstitute for Research on Mental Retardation and Brain Aging, Troina (EN), Italy

J-L Guéant, R-M Guéant-Rodríguez, P Gérard, Cellular and Molecular Pathology in Nutrition, EMI-INSERM 0014, Faculté de Médecine,

Vandoeuvre lès Nancy, France

A Romano, Department of Internal Medicine and Geriatrics, UCSC, Cl Columbus, Rome, Italy

Competing interests: none declared

*The four first authors contributed equally to this article

\section{REFERENCES}

1 Lemaire-Adkins R, Radke K, Hunt PA. Lack of check-point control at the metaphase/anaphase transition: a mechanism of meiotic non-disjunction in mammalian females. J Cell Biol 1997; 139:1611-19.

2 Gericke GS, Hesseling PB, Brink S, et al. Leucocyte ultrastructure and folate metabolism in Down's syndrome. S Afr Med J 1977;51:369-74.

3 MacGregor JT, Wehr CM, Hiatt RA, et al. Spontaneous genetic damage in man: evaluation of inter-individual variability, relationship among markers of damage and influence of nutritional status. Mutat Res 1997;377:125-35.

4 Hassold TJ, Burrage LC, Chan ER, et al. Maternal folate polymorphisms and the etiology of human non-disjunction. Am J Hum Genet 2001;69:434-9.

5 Brattström L, Wilcken DE, Ohrvik J, et al. Common methylenetetrahydrofolate reductase gene mutation leads to hyperhomocysteinemia but not to vascular disease: the result of a meta-analysis. Circulation 1998:98:2520-6.

6 Ueland PM, Hustad S, Schneede J, et al. Biological and clinical implications of the MTHFR C677T polymorphism. Trends Pharmacol Sci 2001;22:195-201.

7 Hyman BT, West HL, Rebeck GW, et al. Quantitative analysis of senile plaques in Alzheimer disease: observation of log-normal size distribution and molecular epidemiology of differences associated with apolipoprotein $E$ genotype and trisomy 21 (Down syndrome). Proc Natl Acad Sci U S A 1995;92:3586-90.

8 Corder EH, Saunders AM, Strittmatter WJ, et al. Gene dose of Apolipoprotein E type 4 allele and the risk of Alzheimer's disease in late-onset families. Science 1993;261:921-3.

9 Bell IR, Edman JS, Selhub J, et al. Plasma homocysteine in vascular disease and in nonvascular dementia of depressed elderly people. Acta Psychiatr Scand 1992;86:386-90.

10 Riggs KM, Spiro A, Tucker K, et al. Relations of vitamin B-12, vitamin B-6, folate, and homocysteine to cognitive performance in the Normative Aging Study. Am J Clin Nutr 1996;63:306-14

11 McCaddon A, Davies G, Hudson P, et al. Total serum homocysteine in senile dementia of Alzheimer type. Int J Geriatr Psychiatry 1998;13:235-9.

12 Clarke R, Smith AD, Jobst KA, et al. Folate, vitamin B12, and serum total homocysteine levels in confirmed Alzheimer disease. Arch Neurol 1998;55:1449-55.

13 Clarke R, Smith AD, Jobst KA, et al. ACE, MTHFR, factor V Leiden, and APOE polymorphisms in patients with vascular and Alzheimer's dementia. Stroke 1998;29:1401-4.

14 Pollak RD, Pollak A, Idelson M, et al. The C677T mutation in the methylenetetrahydrofolate reductase (MTHFR) gene and vascular dementia. J Am Geriatr Soc 2000;48:664-8.
15 Nishiyama M, Kato Y, Hashimoto M, et al. Apolipoprotein E, methylenetetrahydrofolate reductase (MTHFR) mutation and the risk of senile dementia - an epidemiological study using the polymerase chain reaction (PCR) method. J Epidemiol 2000;10:163-72.

16 Miller JW, Green R, Ramos MI, et al. Homocysteine and cognitive function in the Sacramento Area Latino Study on Aging. Am J Clin Nutr 2003;78:441-7.

17 Nilsson K, Gustafson L, Hultberg B. The plasma homocysteine concentration is better than that of serum methylmalonic acid as a marker for sociopsychological performance in a psychogeriatric population. Clin Chem 2000;46:691-6.

18 Bosco P, Gueant-Rodriguez RM, Anello G, et al. In Sicily, methionine synthase (MTR) $2756(A \rightarrow G)$ polymorphism, double heterozygosity methionine synthase $2756 \mathrm{AG} /$ methionine synthase reductase (MTRR) $66 \mathrm{AG}$ and elevated homocysteinemia are three risk factors for having a child with Down syndrome. Am J Med Genet 2003;121:219-24.

19 James SJ, Pogribna M, Pogribny IP, et al. Abnormal folate metabolism and methylenetetrahydrofolate reductase (MTHFR) gene may be maternal risk factors for Down syndrome. Am J Clin Nutr 1999;70:495-501.

20 Wechsler D. Wechsler Adult Intelligence Scale Revised (WAIS R). San Antonio, TX, USA: Psychological Corporation, 1981.

21 Wechsler D. Wechsler Intelligence Scale for Children Revised (WISC R). San Antonio, TX, USA: Psychological Corporation, 1992.

22 American Psychiatric Association. Diagnostic and statistical manual of mental disorders, 4th edn, Text Revision. Washington, DC: American Psychiatric Association, 2000:41-9.

23 Namour F, Olivier J, Abdelmouttaleb I, et al. Transcobalamin codon 259 polymorphism in HT-29 and Caco-2 cells and in Caucasians: relation to transcobalamin and homocysteine concentration in blood. Blood 2001;97:1092-8.

24 Bosco P, Guéant-Rodríguez RM, Anello G, et al. Association of IL-1 RN 2* allele and methionine synthase 2756 AA genotype with dementia severity of sporadic Alzheimer's disease. J Neurol Neurosurg Psychiatry 2004:75:1036-8.

25 Pogribna M, Melnyk S, Pogribny I, et al. Homocysteine metabolism in children with Down syndrome. Am J Hum Genet 2001;69:88-95.

26 Lejeune J, Rethore MO, de Blois MC, et al. Metabolism of monocarbons and trisomy 21: sensitivity to methotrexate. Ann Genet 1986;29:16-19.

27 Ueland PM, Refsum H, Christensen B. Methotrexate sensitivity in Down's syndrome: a hypothesis. Cancer Chemother Pharmacol 1990;25:384-6.

28 Chadefaux B, Ceballos I, Hamet M, et al. Is the absence of atheroma in Down syndrome due to the decreased homocysteine levels? Lancet 1988;2:741.

29 Namour F, Gueant JL. Transcobalamin polymorphism and homocysteine concentration in aging. Blood 2001;98:3498-9.

30 McCaddon A, Blennow K, Hudson P, et al. Transcobalamin polymorphism and homocysteine. Blood 2001;98:3497-8.

31 Miller JW, Ramos MI, Garrod MG, et al. Transcobalamin II 775 G>C polymorphism and indices of vitamin B12 status in healthy older adults. Blood 2002; 100:718-20.

32 Tassone F, Lucas R, Slavov D, et al. Gene expression relevant to Down syndrome: problems and approaches. J Neural Transm 1999;57:179-95.

33 Hattori M, Fujiyama A, Taylor TDK. Chromosome 21 mapping and sequencing consortium. The DNA sequence of human chromosome 21. Nature 2000:405:311-19.

34 Friso S, Choi SW, Girelli D, et al. A common mutation in the 5,10methylenetetrahydrofolate reductase gene affects genomic DNA methylation through an interaction in the folate status. Proc Natl Acad Sci U S A 2002:99:5606-11.

35 Castro R, Rivera I, Struys EA, et al. Increased homocysteine and Sadenosylhomocysteine concentrations and DNA hypomethylation in vascular disease. Clin Chem 2003;49:1292-6.

36 Seshadri S, Beiser A, Selhub J, et al. Plasma homocysteine as a risk factor for dementia and Alzheimer's disease. N Engl J Med 2002;346:476-83.

37 Mattson MP, Shea TB. Folate and homocysteine metabolism in neural plasticity and neurodegenerative disorders. Trends Neurosci 2003;26: 137-46.

38 Drouet B, Fifre A, Pincon-Raymond $M$, et al. ApoE protects cortical neurones against neurotoxicity induced by the non-fibrillar $C$-terminal domain of the amyloid- $\beta$ peptide. J Neurochem 2001;76:117-27

39 Anello G, Gueant-Rodriguez RM, Bosco P, et al. Homocysteine and methylenetetrahydrofolate reductase polymorphism in Alzheimer's disease. NeuroReport 2004;15:859-61. 